\title{
Evaluating Secondary Input Devices to Support an Automotive Touchscreen HMI: A Cross-Cultural Simulator Study Conducted in the UK and China
}

\author{
David R Large ${ }^{1}$, Gary Burnett ${ }^{1}$, Elizabeth Crundall ${ }^{1}$, Glyn Lawson ${ }^{1}$, Lee Skrypchuk ${ }^{2}$, Alex Mouzakitis ${ }^{2}$ \\ ${ }^{1}$ Human Factors Research Group, Faculty of Engineering, University of Nottingham, UK \\ ${ }^{2}$ Jaguar Land Rover Research, International Digital Laboratory, Coventry, UK
}

\begin{abstract}
Touchscreen Human-Machine Interfaces (HMIs) are a well-established and popular choice to provide the primary control interface between driver and vehicle, yet inherently demand some visual attention. Employing a secondary device with the touchscreen may reduce the demand but there is some debate about which device is most suitable, with current manufacturers favouring different solutions and applying these internationally. We present an empirical driving simulator study, conducted in the UK and China, in which 48 participants undertook typical in-vehicle tasks utilising either a touchscreen, rotary-controller, steering-wheel-controls or touchpad. In both the UK and China, the touchscreen was the most preferred/least demanding to use, and the touchpad least preferred/most demanding, whereas the rotary-controller was generally favoured by UK drivers and steering-wheel-controls were more popular in China. Chinese drivers were more excited by the novelty of the technology, and spent more time attending to the devices while driving, leading to an increase in off-road glance time and a corresponding detriment to vehicle control. Even so, Chinese drivers rated devices as easier-to-use while driving, and felt that they interfered less with their driving performance, compared to their UK counterparts. Results suggest that the most effective solution (to maximise performance/acceptance, while minimising visual demand) is to maintain the touchscreen as the primary control interface (e.g. for top-level tasks), and supplement this with a secondary device that is only enabled for certain actions; moreover, different devices may be employed in different cultural markets. Further work is required to explore these recommendations in greater depth (e.g. during extended or real-world testing), and to validate the findings and approach in other cultural contexts.
\end{abstract}

\section{Keywords:}

Touchscreen; rotary controller; steering wheel control; touchpad; visual demand; preferences; driving performance; workload; character recognition; culture; HMI; UK; China; driver acceptance 


\section{INTRODUCTION}

Touchscreens are increasingly commonplace as the primary display and control interface in vehicles. They can be more effective for some tasks (e.g. simple menu selection), and attract more positive responses from drivers, compared to other in-vehicle devices (Burnett et al., 2011). Nevertheless, touchscreens inherently require some visual attention, due in part to designers' slavish adherence to skeuomorphic interface elements to reflect previously physical buttons: the absence of genuine tactile cues associated with such 'buttons' means that drivers must visually sample the interface to locate control elements and view task progress. As a result, common in-car secondary controls and infotainment services could demand too much visual attention if presented on a touchscreen-centric system. This can result in deleterious effects on driving performance and vehicle control, thereby elevating the risk to drivers and other road users (NHTSA, 2013).

Consequently, there has been significant research effort devoted to mitigating the visual (and manual) demand elicited by such devices. This has taken a number of guises, including designing interactive on-screen elements to minimise visual demand (Eren et al., 2015a), comparing different list-scrolling techniques (Large et al., 2013), and identifying simple, intuitive 'short-cut' gestures (Burnett et al., 2013; Eren et al., 2015b). Other novel techniques, such as expanding or predicting touchscreen targets based on drivers' mid-air finger proximity have also been explored (e.g. Aslan et al., 2015; Ahmad et al., 2015).

An alternative approach explored here is to employ a secondary input device to work in unison with the touchscreen to ease the visual/manual burden of interactions, while maintaining the overall appeal and flexibility of the touchscreen. Current popular examples of such secondary devices include, rotary controllers, steering wheel controls and touchpads. The novel 'combined' use of input and output devices has the potential to improve the usability of in-vehicle secondary activities, enabling a safer and more fluid integration with the primary driving task, and provides the necessary flexibility and adaptability for users who may differ considerably in their attitude, ability, experience, and expectations towards technology. In addition, by utilising a physically distinct secondary device, drivers would be able to locate the control without vision. Moreover, by reintroducing haptic cues during operation, such as 'clicking' as the user moves through options in a list (as one might expect with traditional buttons, knobs and switches), aspects of the interaction could be completed without vision. It is worth noting that although attempts have been made to add haptic cues to touchscreens, for example to simulate the depression of a button (Pitts et al., 2012), such 'soft' buttons have received limited success and may ultimately fail to deliver fully the complex cutaneous sensations associated with traditional, physical knobs and buttons (Zwahlen et al., 1988).

A further advantage to using a 'vision-free' secondary input device, is that it may be positioned in the most appropriate location based on anthropometrical considerations. This is likely to reduce fatigue effects during operation, and potentially eliminate handedness problems. Furthermore, a physical device (particularly if located in a more favourable location where the driver's arm is supported) is also likely to lead to better operational accuracy compared to a touchscreen alone, particularly when the latter is located in the upper centre-console with no arm support (Porter et al., 2005). 


\section{Driver Acceptance and Culture}

The successful introduction of any new driver-centric technology in vehicles is not only contingent on its technical proficiency (particularly where it forms part of an integrated solution), but also on the degree to which drivers accept and use the devices as intended. Acceptance is fundamentally based on the user's understanding of and attitudes towards the system, and its potential to deliver the intended benefits. These factors are particularly relevant if drivers are expected to interact with multiple devices to achieve a single in-vehicle task, or are presented with several different methods to achieve the same goal (which is often the case with current in-vehicle HMIs). In an automotive domain, acceptance has been defined as: "the degree to which an individual intends to use a system and, when available, incorporates the system in his/her driving" Adell (2010, p.477) - highlighting the importance of 'using' the system - and is generally captured by recording the user's subjective evaluation of their satisfaction with the experience of interaction and use. Acceptance is complimented by acceptability, which is defined as the "prospective judgment of measures to be introduced in the future" (Schade and Schlag, 2003, p. 47), or how much a system is 'liked' (Jamson, 2010). Although acceptance and acceptability appear as lucid concepts within the literature, they are frequently both used to refer to general attitudes towards new technology, and during the current study, we aim to explore aspects associated with both constructs.

A further consideration is that, given the global nature of the automotive market, where vehicles and their associated HMIs and technology may be deployed across a number of different, culturallydiverse markets, drivers' acceptance (and acceptability) of novel, in-vehicle technology may also be affected by a local cultural perspective. Moreover, 'poorly designed' products or technology that appear to contravene a culture's core values can lead to difficulty comprehending their role or purpose, and may result in difficulties during use - such items are consequently less likely to be accepted, and may even upset or offend prospective users (see: Allison, 1995; Gibson, 1993).

In a cultural driving context, there is some evidence that questionnaires and 'paper-based' approaches have been employed to elicit culturally-relevant user requirements and preferences, which could be applied to devices and interfaces intended for deployment within vehicles (e.g. Large et al., 2017; Olaverri-Monreal and Bengler, 2011; Young et al., 2012). Nevertheless, self-reported questionnaires are notoriously contextual and responses may be subject to biases, particularly in a cross-cultural context, where one of the cultural groups being studied could be prone to a certain response-set bias (e.g. extreme, neutral, agree or disagree behaviour) (Kerlinger, 1973). It has also been suggested that in some cultures, such as China, respondents might favour certain numbers on a scale that are considered lucky, and conversely avoid those that are deemed unlucky (Roy et al., 2001). Questionnaire respondents may also attempt to portray themselves in a more favourable light when questioned (Couch and Keniston, 1960). For example, drivers from Western, industrialised cultures tend to regard themselves as more skilful than the "average driver" (Goszczyńska and Rosłan, 1989), and often report lower levels of driving violations (e.g. speeding) and poor driving behaviour (e.g. tailgaiting) than the evidence shows (Özkan et al., 2006.). Consequently, it can be difficult to develop any practical design guidance from questionnaire responses alone.

Several empirical studies have attempted to identify cultural differences in user preferences for invehicle technology and integrate these within designs. For example, Chinese drivers responded 
unfavourably when presented with an experimental prototype navigation system structured according to German users' mental models of a navigation system, and vice versa (Knapp, 2007). There is also evidence to suggest that drivers in China considered the usefulness of in-vehicle devices to be of greatest importance, whereas Indonesians considered simplicity of use to be paramount. Conversely, Australian drivers were motivated by the aesthetics of the design (Khaslavsky, 1998). Elsewhere, Chinese drivers expressed preferences towards flat information hierarchies and clearer information layouts that provide greater transparency and freedom, and were less concerned with the ability to customise an interface than Australian drivers (Young et al., 2012). It is noteworthy that the some of the latter results are in contrast to earlier 'culture' research (e.g. Hofstede, 1995; Marcus, 2003). Young et al. (2012) cite rapid changes within Chinese society/culture in terms of demographics and attitude over the last ten years - in particular, shifts in class and increases in salaries - for the differences they encountered, highlighting the potential fluidity of cultural preferences.

\section{Overview and Aims of Study}

There has been significant research effort devoted to different input devices and HMIs in vehicles, and a corpus of literature exists. This appears to have encouraged current automotive manufacturers to adopt a number of different in-vehicle HMI solutions (with exemplars such as the BMW iDrive rotary controller and Audi MMI touchpad). However, there is very little published research that considers the potential effects, benefits and user requirements/preferences, when combining devices. Moreover, given the global nature of the automotive market, whereby technological solutions may be deployed across several international markets, there is a further need to consider these factors at a country or cultural level. Current efforts at cross-cultural differentiation appear to be limited to the perfunctory translation of written elements to the native language and/or the potential mirroring or relocation of hardware and/or software components to take account of different driving positions (i.e. left versus right-hand drive vehicles).

The current driving simulator study therefore aims to first explore the effects of using different input devices on driver distraction and performance, and elicit preferences for a secondary input device, or devices, that could be used in combination with a touchscreen. The second aim of the study is to investigate differences in drivers' performance, behaviour and attitudes towards the devices ('acceptance') at a country level (between the UK and China), which we interpret from a cultural perspective. The UK and China were chosen because their culture, language and driving environment differ substantially from one another (Young et al., 2012). Moreover, both nations represent significant international markets for many automotive manufacturers. The study was initially conducted in the UK at the University of Nottingham by a British research team. Subsequent testing was conducted in China at the University of Nottingham Ningbo campus (UNNC) by the same research team, but with the support and guidance of local collaborators.

\section{METHOD}

\section{Participants}

Forty-eight experienced drivers took part in the study (twenty-four in the UK and twenty-four in China). All participants were 'culturally immersed' in their native country, meaning, for example, that 
in the UK, all participants were British nationals (or held long-term residency in the UK), held UK driving licenses and predominantly drove in the UK. Equivalent statements were true for those taking part in China. However, an additional requirement for Chinese participants was that they had not been exposed extensively to Western culture (for example, they did not study or work at the University of Nottingham Ningbo campus, where the investigation took place).

Participants in the UK were self-selecting volunteers who responded to advertisements placed on-line and around the University campus, and were reimbursed with shopping vouchers in lieu of their time. Chinese participants were selected by a local recruitment agency, to ensure that common demographics - age, gender, handedness, driving experience - were matched as closely as possible between the two groups, and that other requirements (e.g. cultural immersion) were adhered to. Chinese participants were paid to take part in the study.

In both nations, there were eleven male and thirteen female drivers. UK participants' mean age was 32, with ages ranging from 21 to 51 years. Twenty of the UK participants were right-handed and four, left-handed. Chinese participants ranged from 22 to 52 years old, with a mean age of 32 years. Twentyone participants in China were right-handed and three, left-handed. All participants were licensed and active drivers. In the UK, participants' mean number of years driving was 12.5 (range 4-31 years); mean current annual mileage was 7495 miles and range, 500 to 20000. In China, participants had, on average, been driving for 6.3 years (range: 2 to 20 years) and their mean current annual mileage was 11700 (range: 4000 to 20000 miles). The aim when recruiting participants was to include a broad range of drivers (age, driving experience, handedness, gender). However, these factors were not controlled as independent variables and therefore not considered during the analysis. The study had been approved by the University of Nottingham Faculty of Engineering ethics committee to be undertaken in the UK and UNNC China. All participants provided written informed consent before taking part.

\section{Apparatus}

The simulated driving environment was created using STISIM (v2) software and comprised a straight, three-lane motorway (or 'highway'). For authenticity, road signage (consistent with local language and 'rules of the road'), geo-typical roadside scenery, and on and off slip-roads were included; both sides of the carriageway were populated with moderate levels of traffic.

Participants interacted with the driving scenario using a Logitech G27 force feedback steering wheel and pedals (both simulators were configured with automatic gear selection, and therefore no gearchange shifter was required). In the UK, the Logitech controllers were integrated within the front half of a static 2001 right-hand drive Honda Civic SE car, replacing the original car controls (Figure 1). The simulator buck was positioned within a curved screen providing approximately $270^{\circ}$ viewing angle, with the driving scenario projected onto the screen using three overhead projectors. Rear views were relayed using video cameras to small LCD displays appropriately located to replicate right and left wing mirrors, and to a screen situated behind the car, which could be seen from the driver's perspective using the existing rear-view mirror. Road and engine speed data were extracted from the STISIM software using a bespoke Java application and re-presented as an analogue speedometer and tachometer, respectively, on an eight-inch LCD display fitted into the existing car instrument panel to mimic the car dashboard. 
It was not practicable to transport the full UK driving simulator to China for the study. Nevertheless, the salient aspects were closely adhered to. Namely, the same Logitech G27 driving controls (steering wheel and pedals) were used in China, but were mounted onto a support positioned in front of a single screen rather than within a simulator buck (Figure 2). An equivalent driving scenario was projected onto a flat screen, positioned directly in front of participants, using an overhead projector. Mirror displays (right, left and rear-view) and the driver's dashboard (speedometer, tachometer) were embedded within the projected image, utilising existing STISIM software functionality, so that they appeared at appropriate locations from the driver's perspective.

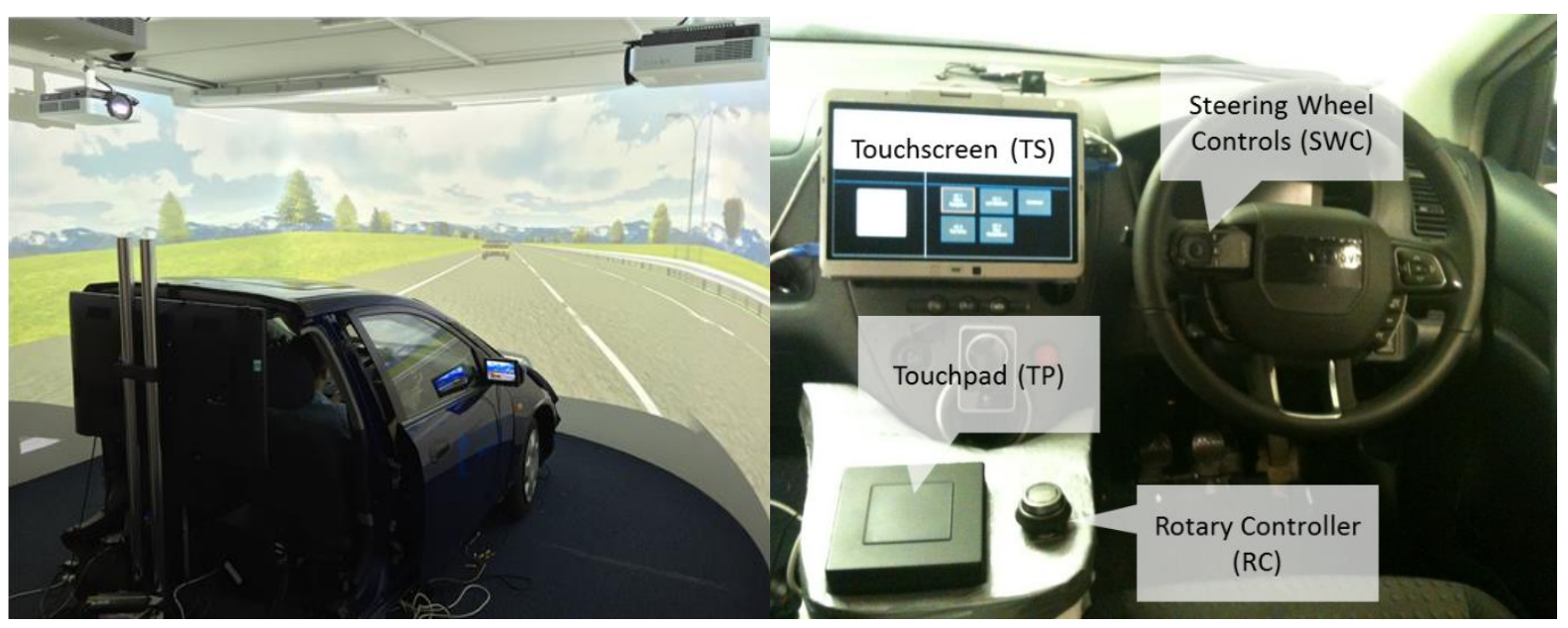

Figure 1: Experimental set-up in the UK, showing medium-fidelity driving simulator and device locations

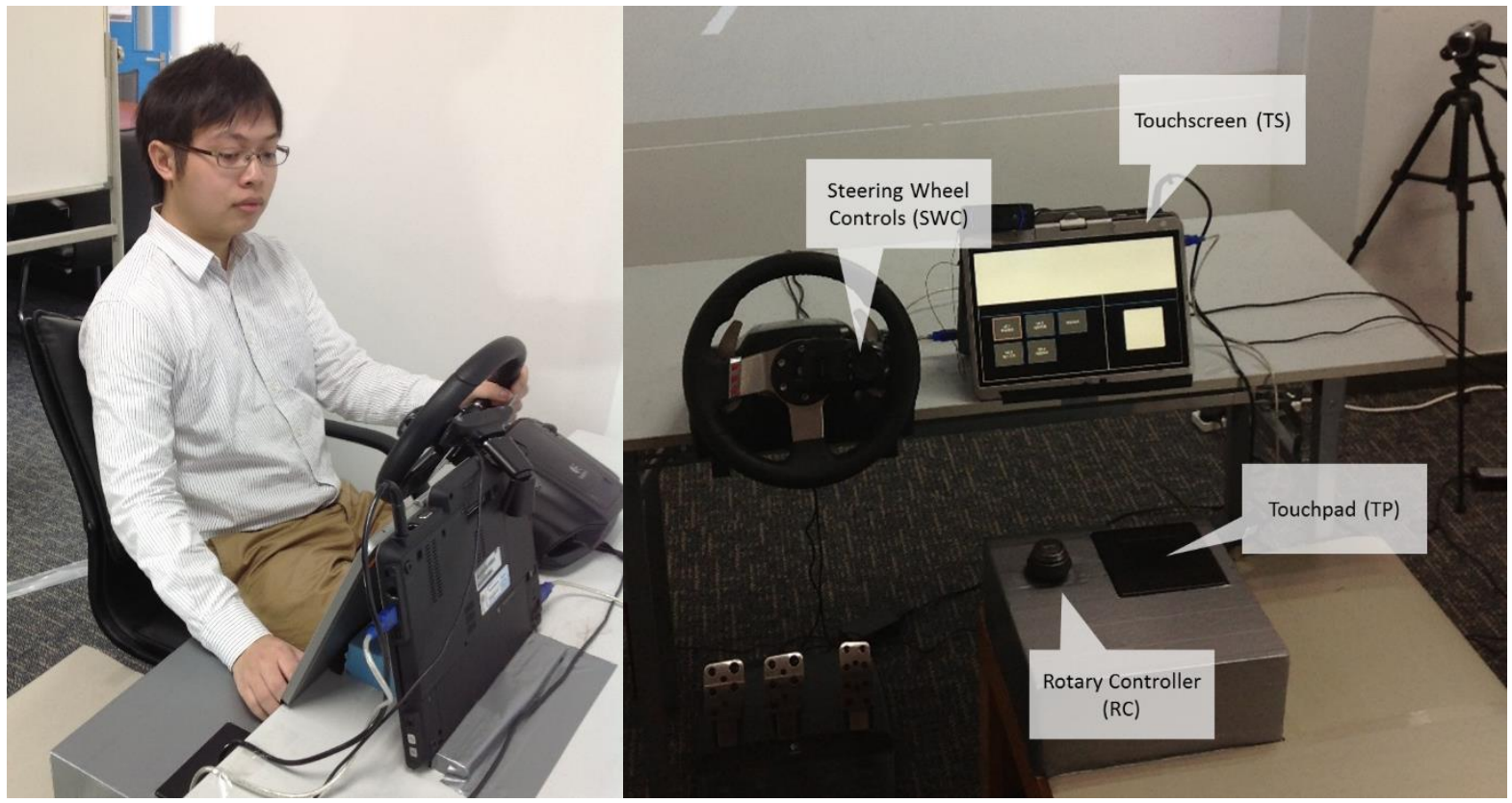

Figure 2: Experimental set-up In China, showing desktop driving simulator and device locations. Note: mirror displays and dashboard were embedded in the projected image. 
The touchscreen (an HP EliteBook 2740p tablet computer) and input devices were located in identical positions with respect to the driver in both simulators. The UK simulator resembled a right-hand drive car and thus the centre console and devices were to the left. In China, the simulator emulated a lefthand drive vehicle. Consequently, the locations of all devices under examination were mirrored, e.g. the steering wheel controls were embedded on the left-hand spine of the steering wheel in UK and the right-hand spine in China, and the driving scenario was modified to allow participants to drive on the left in the UK scenario and on the right in China.

In both locations, vehicle control data - notably road speed, lateral lane position and headway - were recorded using the simulation software. Audio-visual recordings of the participant, devices and driving scenario were also made during the experiment using cameras strategically positioned for nonobtrusive data capture. In China, the video recordings were used to determine participants' eye movements on a frame-by-frame basis. In the UK, an additional ETG (Eye Tracking Glasses) system from SensoMotoric Instruments (SMI) was used to record participants' visual behaviour. In both situations, the obtained eye glance data were analysed on a frame-by-frame basis in line with the ISO standard (2014).

\section{Experimental Design and Procedure}

The 'car following' paradigm was adopted as the primary driving task in both countries (Brookhuis, et al., 1994) - a yellow car appeared on the motorway at the start of the simulated driving scenario and continued at a constant speed of $65 \mathrm{mph}$. Participants were instructed to join the motorway, follow the lead car at a distance that they deemed to be safe and appropriate, and drive as they would in the real world (i.e. obeying the 'rules-of-the-road' and prioritising the driving task).

While following the lead vehicle ('the primary task'), participants were asked to undertake four invehicle tasks ('the secondary task'). In both nations, participants were provided with a verbal instruction to begin each task, but were specifically instructed that they should only undertake the secondary task if and when they felt it was safe to do so. Performance measures were subsequently determined from the time that each participant began the interaction, and not the time that the instruction was delivered.

The secondary tasks were completed using each of four different devices/interfaces. The devices were selected in collaboration with a major UK vehicle manufacturer, and comprised: a rotary controller, wireless mouse, touchpad and touchscreen. All devices were designed to be used in conjunction with a touchscreen, located in the centre console, and were positioned in typical or expected locations within each 'vehicle' (Figures 1 and 2). The touchscreen acted as both a display (to provide feedback for the rotary controller, wireless mouse and touch pad), and as an input device in its own right during the touchscreen-only condition. The wireless mouse (a Sony Vaio Bluetooth laser mouse, model VGPBMS80) was embedded within the left (UK) or right (China) spindle of the steering wheel to mimic steering wheel controls, and is hereafter referred to as such. The touchpad and rotary controller were located at the base of the centre console (between the driver and passenger seats), and an arm rest was provided to support participants' arms when using these devices. Further details of the devices and their native interaction techniques are provided in Table 1. 
Table 1: Devices investigated during study, with details of native interaction techniques

\begin{tabular}{|c|l|}
\hline Device & \multicolumn{1}{c|}{ Interaction Techniques } \\
\hline Rotary Controller (rotary) & $\begin{array}{l}\text { Rotary input in addition to 4-way 'joystick' and button } \\
\text { presses. }\end{array}$ \\
\hline $\begin{array}{c}\text { Steering Wheel Controls (steering } \\
\text { controls) }\end{array}$ & $\begin{array}{l}\text { Four-way directional control in addition to optical swipe } \\
\text { and button press inputs. }\end{array}$ \\
\hline $\begin{array}{c}\text { Touchpad } \\
\text { Touchscreen }\end{array}$ & $\begin{array}{l}\text { Four-way swipe, button press and character/gesture } \\
\text { recognition, using fingertip input. }\end{array}$ \\
\hline & $\begin{array}{l}\text { Single and multiple finger input (touch, drag, pinch-to- } \\
\text { zoom etc.). }\end{array}$ \\
\hline
\end{tabular}

The four tasks undertaken by drivers were representative of in-vehicle driving-related activities and were enabled using a bespoke, test interface (Figure 3). Tasks comprised: text entry (text), menu navigation (menu), map manipulation (map) and list selection (list) (Table 2). Text entry and menu navigation tasks were identical between countries. For the list selection task, the target music track differed between countries (to correspond with common local music preferences), although the target item in each country was located at the same physical position in their respective list, and therefore required the same number and type of interactions to locate, highlight and select. The map used during the map task differed between countries to ensure it was geographically relevant, but the task itself was equivalent in so far as the physical manipulation and device inputs required. For all tasks, any text-based content (e.g. button labels, music items for the list search task, etc.), was presented in the appropriate, native language.

To complete each task, participants were required to use the native input techniques for each device. This ensured that participants were able to experience the full functionality of all devices, thereby allowing more robust judgements and comparisons to be made, particularly regarding preferences and relative performance. For example, to move through a list, participants were required to swipe the touchscreen and touchpad, rotate the rotary controller, or press 'up' and 'down' buttons on the steering wheel controller. To enter alphanumeric characters, participants were provided with an onscreen keyboard for the touchscreen, rotary and steering controls, but were required to 'write' each character individually with their index finger, when using the touchpad.

Prior to testing, participants received full training and guidance for each device, and for all tasks, until they were deemed to be competent by the researcher (in line with common testing procedure (e.g. NHTSA, 2013). During testing, participants completed all four tasks using each device while driving, providing subjective feedback between devices and drives. Device and task order were counterbalanced to avoid learning effects. After testing all four devices, participants undertook a fifth, 
'free-choice' drive, in which they were able to choose their preferred device (or combination of devices) to complete each of the four tasks.

Table 2: Tasks undertaken during study

\begin{tabular}{|c|l|}
\hline \multicolumn{1}{|c|}{ Task } & \multicolumn{1}{c|}{ Description } \\
\hline Menu Navigation (menu) & $\begin{array}{l}\text { Move through four different menu configurations by selecting } \\
\text { the option highlighted by an ' } X \text { '. }\end{array}$ \\
\hline List Selection (list) & $\begin{array}{l}\text { Search and select a specified music track from the media player } \\
\text { (required negotiation of a 'long', multiple-screen, list). }\end{array}$ \\
\hline Text Entry (text) & $\begin{array}{l}\text { Enter a specified phone number using the telephone interface, } \\
\text { and select 'Call'. }\end{array}$ \\
\hline Map Manipulation (map) & $\begin{array}{l}\text { Select 'pan' and/or 'zoom' buttons to view and traverse a route } \\
\text { highlighted on the map. }\end{array}$ \\
\hline
\end{tabular}
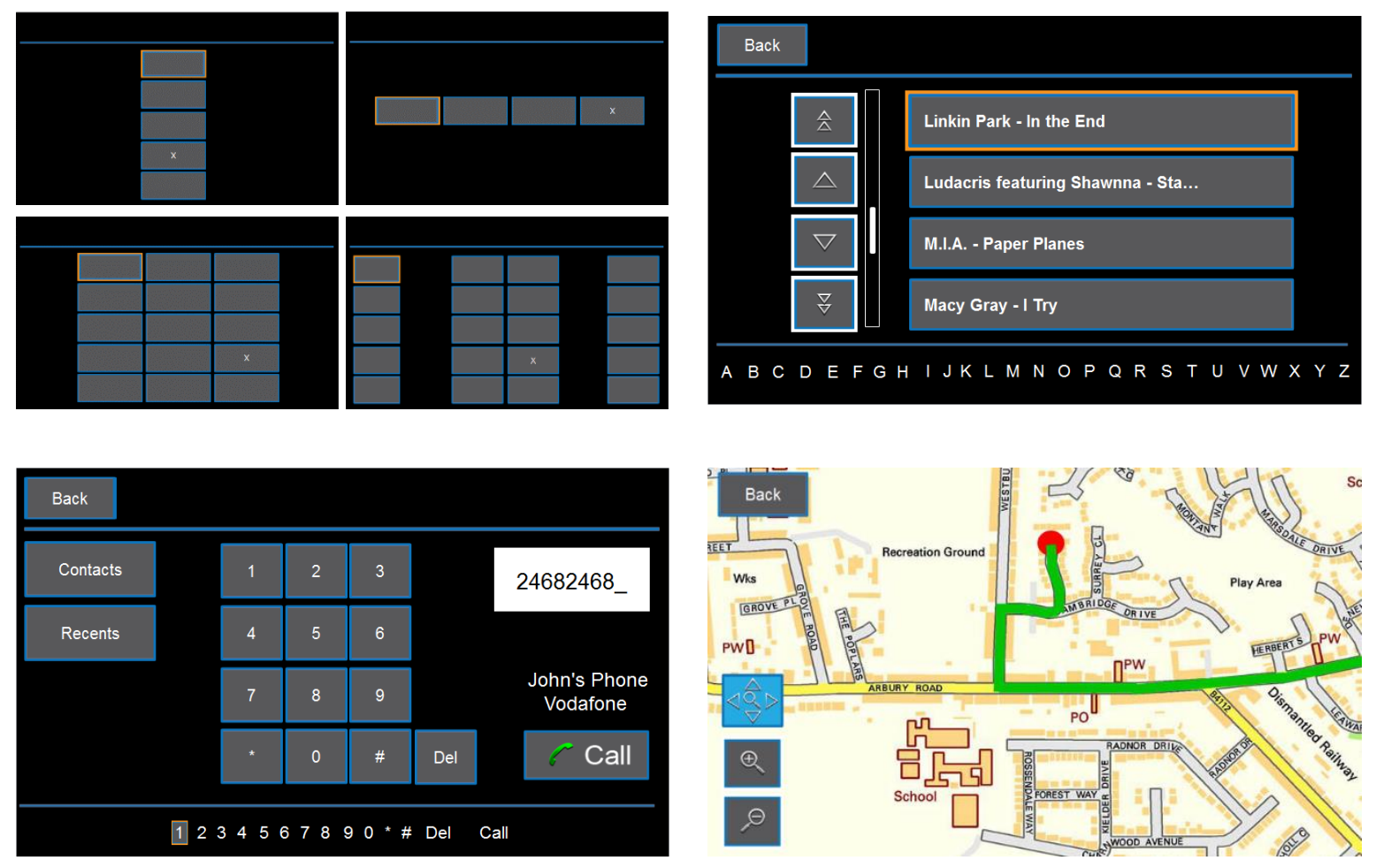

Figure 3. UK version of bespoke test interface used during study, showing (clockwise from top left): Menu Navigation, List Selection, Map Manipulation and Text Entry tasks. 


\section{Measures}

The following measures were captured and reported:

- Task Performance, measured by secondary task time recorded from the bespoke interface/touchscreen from the commencement of the interaction.

- Visual Behaviour - total glance time (TGT), mean glance duration (MGD) and number of glances (NG), as defined by NHTSA (2013). Glances were classified as 'on' or 'off-road'. Offroad glances reflected visual attention directed at both the device for control and the touchscreen for feedback.

- Driving Performance - speed, lane keeping, headway from lead vehicle, captured from the STISIM simulation computer.

- Workload - NASA-TLX mean workload rating (Hart and Staveland, 1988).

- Emotional Response - ratings of 'dominance', 'arousal' and 'control', obtained from the SelfAssessment Manikin (SAM) questionnaire (Bradley and Lang, 1994).

- Subjective Ratings and Preferences - ease of use while driving, interference with driving task, device preferences/liking etc., captured using a bespoke questionnaire with 7-point Likert scales (see: Appendix for UK version).

Participants provided subjective feedback (NASA-TLX, SAM and Preferences) after each task and for each device when stationary, and after completing all four tasks using each device while driving. In the UK, participants recorded their own ratings by completing a written questionnaire while seated in the simulator buck. In China, the questions (which had been translated into Chinese) were delivered verbally by a local collaborator immediately after each task had been completed using each device (both while stationary and driving); the collaborator subsequently recorded participants' responses. The different approach used to elicit subjective opinions and ratings in China was adopted based on the advice of our local collaborator as a more effective method to yield reliable results (see: Stening and Zhang, 2007). Subjective ratings (workload, emotional response, preferences etc.) were collectively interpreted as an indicator of 'driver acceptance' in line with current acceptance theory and measurement (see: Horberry et al., 2017).

\section{RESULTS AND ANALYSIS}

Unless otherwise stated, 3-way ANOVAs were conducted to examine the effects of Device (rotary, steering controls, touchpad, touchscreen), Task (menu, list, text, map) and Country (UK, China) on each measure, with post hoc Tukey corrections for multiple comparisons. All figures show standard errors bars, where appropriate.

\section{Task Performance (Secondary Task Time)}

Participants were required to complete each task using each device (i.e. tasks could not be left incomplete), therefore secondary task time was used as a surrogate measure for task performance. There were significant main effects of Device $(\mathrm{F}(3,105)=84.6 ; p<.001)$ and Task $(\mathrm{F}(3,105)=11.7 ; p$ $<.001$ ) on secondary task time, showing that participants took significantly longer to complete tasks 
using the touchpad and were quickest when utilising the touchscreen. In addition, participants took significantly longer to perform the text task than all other tasks. There were also significant interactions between Country and Task $(F 3,105=2.9 ; p<0.05)$, indicating that Chinese drivers took significantly longer to complete menu and list tasks, compared to UK participants, but performance was comparable during text and map tasks. An interaction between Device and Task ( $F 9,315=10.751$; $\mathrm{p}<0.001$ ) indicated that although using the touchscreen was quickest for all tasks, relative performance using the other devices varied considerably (Figure 4).

घP $₫ R C \backsim S W C \unrhd T S$

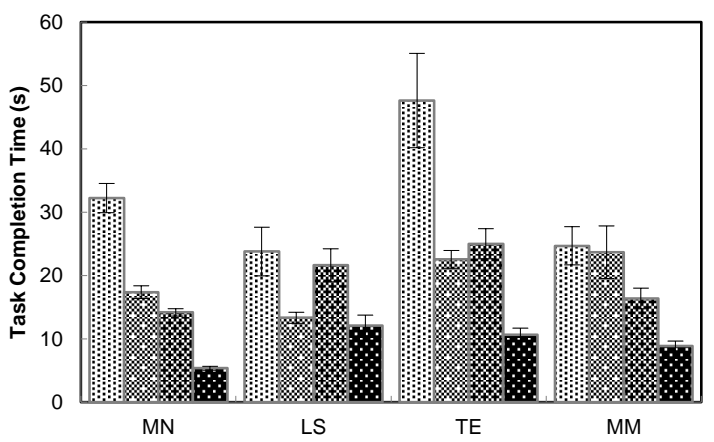

由TP ⿴RC 由SWC -TS

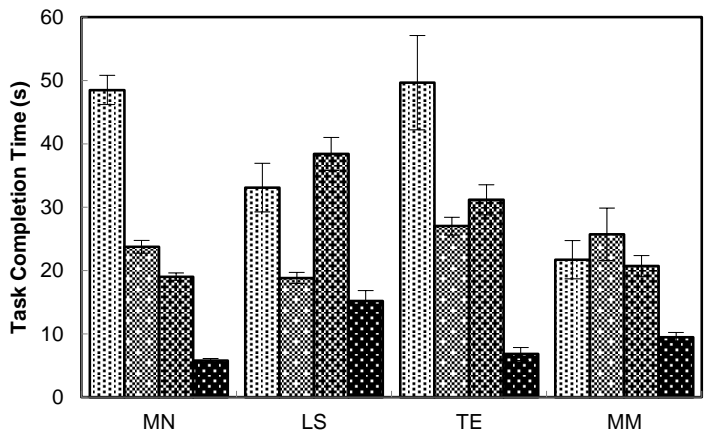

Figure 4: Secondary task times for participants from UK (left) and China (right), with devices (TP = touchpad, RC = rotary controller, SWC = steering wheel controls, $\mathrm{TS}=$ touchscreen) and tasks (MN = menu navigation, $\mathrm{LS}=$ list selection, $\mathrm{TE}=$ text entry, $\mathrm{MM}=$ map manipulation)

\section{Driving Performance (Standard Deviation of Speed, Lane Position and Headway)}

There was a main effect of Device on the standard deviation of speed $(F(3,105)=21.6 ; p<.001)$, with greater variability in speed evident when participants were using the touchpad, compared to all other devices; less speed variability was evident with the touchscreen. There was a significant main effect of Country $(F(1,35)=4.7 ; p<.05)$, with Chinese participants presenting more variability in speed profiles compared to UK participants overall (Figure 5), and a significant interaction between Country and Device $(F(3,105)=3.7 ; p<.05)$. There was also a significant main effect of Task $(F 3,105=3.4$; $p<0.05$ ), indicating that participants' speed profiles were more consistent while undertaking the map task, than during the list and text tasks. In addition, there was a significant interaction between Device and Task (F9,315 = 3.3; $p<0.01)$.

There was also a significant main effect of Device for standard deviation of lane position (SDLP) $(F(3,105)=8.3 ; p<.001)$, showing better lane stability and lateral control when drivers were using the touchscreen compared to other devices, and with the steering controls compared to the touchpad. There was a significant effect of Country $(F(1,35)=33.5 ; p<.001)$, and a significant interaction between Country and Device $(F(3,105)=3.7 ; p<.05)$, for SDLP, showing that Chinese participants were more erratic than UK participants in their lateral lane behaviour (Figure 5).

For headway to the lead vehicle, there was a significant main effect of Device $(F(3,105)=21.2 ; p<$ .001 ), showing that longitudinal control (i.e. standard deviation of headway) was better with the touchscreen than with other devices, and worst for the touchpad. There was a marginal effect of 
Country $(F(1,35)=4.0 ; p=0.055)$, with Chinese participants' displaying more erratic longitudinal behaviour than their UK counterparts (Figure 5 ). There was also a significant interaction between Device and Task $(F(9,315)=1.9 ; p<.05)$.
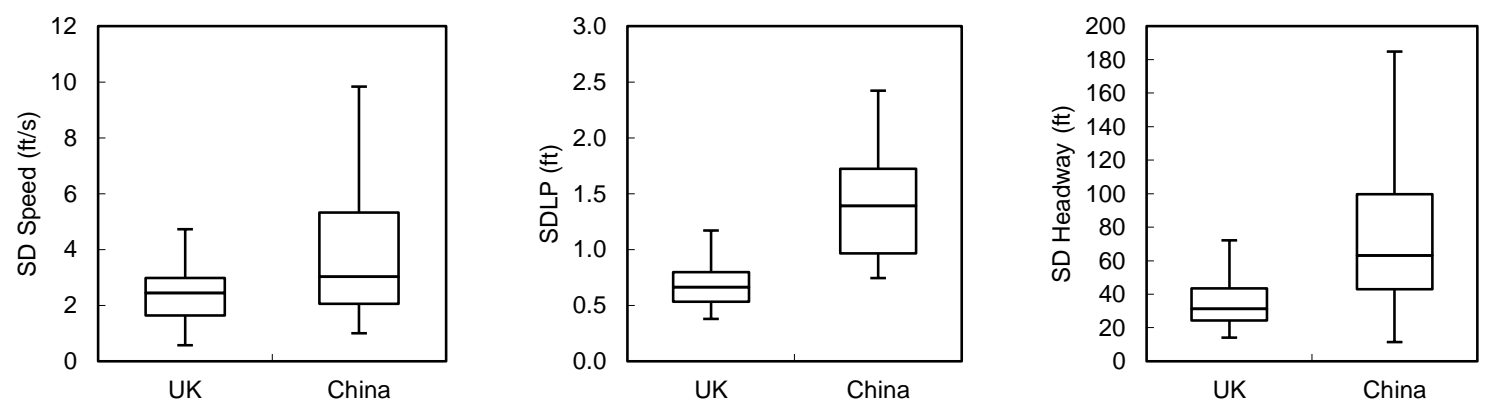

Figure 5: Comparison of driving performance measures (amalgamated for all tasks and devices) between the UK and China, showing speed variability (left), SDLP (centre) and SD headway (right)

\section{Visual Behaviour (Total Glance Time, Mean Glance Duration, Number of Glances)}

Analysis of total glance time (TGT) revealed a significant main effect of Device $(F(1.35)=68.23 ; p<$ $.001)$ and Country $(F(1,35)=13.99 ; p<.01)$, indicating that on average participants spent more time looking off-road when using the touchpad than when using the other devices, and spent less time looking off-road with the touchscreen. In addition, Chinese participants spent more time looking offroad than UK participants while using the devices overall (Figure 6). There was also an interaction between Country and Device $(F(3,105)=4.57 ; p<.01)$, and a three-way interaction between Country, Device and Task $(F(9,315)=2.04 ; p<.05)$, indicating that the total amount of time that Chinese participants spent looking off-road was greater than UK participants for most tasks and devices, except the text task using the touchscreen, during which UK participants' total off-road glances were longer than Chinese participants.

For mean glance duration (MGD), there was a main effect of Device $(F(3,105)=14.5 ; p<.001)$ and Country $(F(1,35)=19.31 ; p<0.001)$, with results indicating that participants made longer off-road glances with the touchscreen than any other device. Chinese participants made significantly longer off-road glances than UK participants (Figure 6). There was also a significant main effect of Task for $\operatorname{MGD}(\mathrm{F93}, 105)=20.67 ; p<.001)$, and an interaction between Device and Task $(\mathrm{F}(9,315)=8.55 ; p<$ .001 ) showing that using the touchscreen resulted in longer off-road glances than other devices in list and text tasks (although there were no differences for menu and map tasks). In addition, the steering controls produced longer glances than touchpad during the text task.

Analysis of the number of off-road glances (NG) revealed a significant main effect of Device $(F(3,105)$ $=128.0 ; p<.001$ ), with participants making fewer off-road glances when using the touchscreen (and more off-road glances with the touchpad) than with any other device. There was no significant main effect of Country for NG (Figure 6). However, there was an interaction between Country and Device $(F(3,105)=2.82 ; p<0.05)$, indicating that Chinese participants made significantly fewer off-road glances than their UK counterparts when using the touchscreen. For NG, there was also a significant main effect of Task $(F(3,105)=10.46 ; p<.001)$, and an interaction between Task and Device $(F(9,315)$ 
$=14.84 ; \mathrm{p}<.001)$, indicating that there were fewer glances associated with the rotary compared to the steering controls for the list task, but more glances associated with the rotary compared to the steering controls for the map task.

There were also significant main effects of Device $(F(3,240)=5.21 ; p=.002)$ and Task $(F(3,240)=$ 2.91; $p=.04$ ) on the number of 'long glances' (i.e. over 2.0-seconds), with significantly more 'long glances' associated with the touchpad compared to all other devices $\left(p_{\max }=.047\right)$.
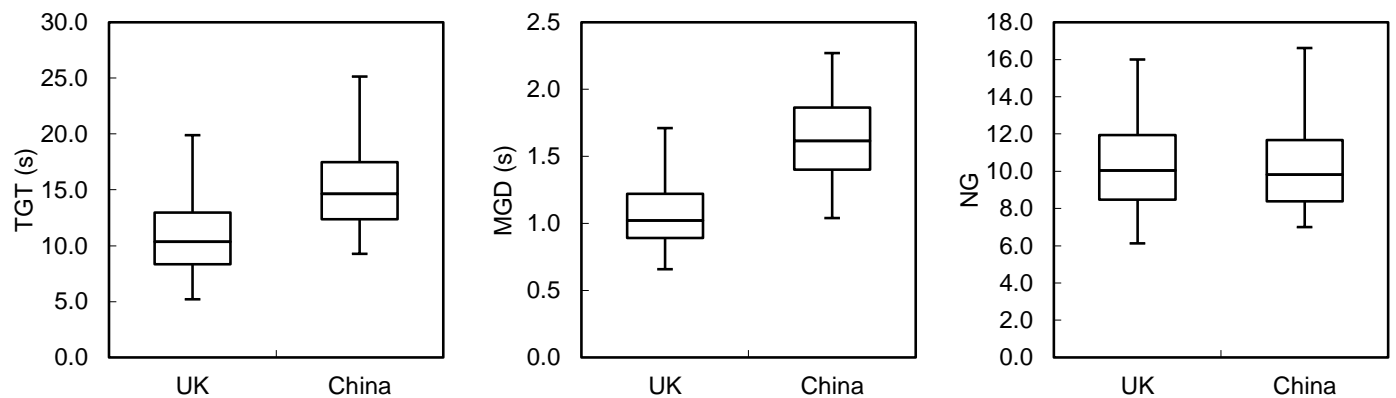

Figure 6: Comparison of visual behaviour measures (amalgamated for all tasks and devices) between the UK and China, showing total glance time (TGT) (left), mean glance duration (MGD) (centre) and number of glances (NG) (right)

\section{Workload (NASA-TLX)}

For NASA-TLX mean workload, there was a significant main effect of Device $(F(3,139)=36.2 ; p<.001)$, indicating that the touchpad was significantly more demanding, and the touchscreen significantly less demanding to use, compared to other devices (Figure 7). In addition, there was a significant interaction between Device and Country $(\mathrm{F}(3,138)=2.91 ; p=.037)$, with Chinese participants rating the rotary controller as significantly more demanding to use than UK participants; ratings for the other devices were comparable between the UK and China.

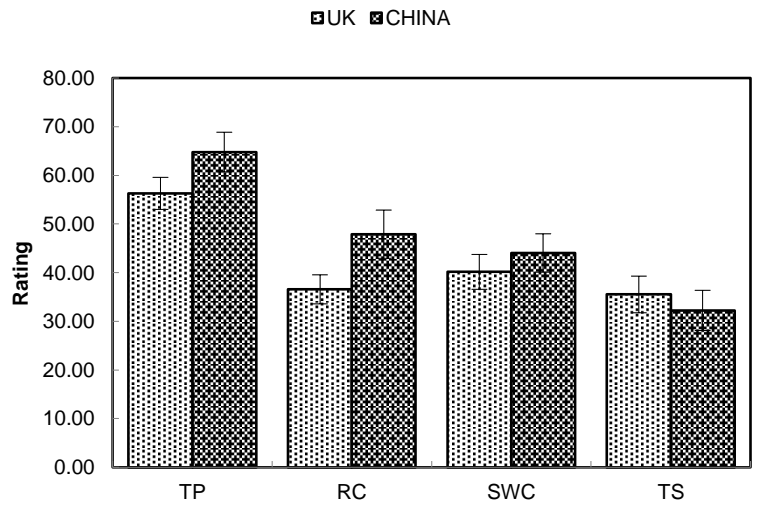

Figure 7: Mean workload for all tasks by device, where TP = touchpad, RC = rotary controller, SWC = steering wheel controls, and TS = touchscreen 


\section{Emotional Response (Self-Assessment Manikin, SAM)}

The SAM questionnaire revealed significant main effects of Device for ratings of Pleasure $(F(3,138)=$ 37.4; $p<.001)$, Arousal $(F(3,138)=10.1 ; p<.001)$ and Dominance $(F(3,138)=37.3 ; p<.001)$, showing that for all three dimensions, the touchpad was rated lowest (least pleasurable, least stimulating and most difficult to control) and the touchscreen received the highest ratings (most pleasurable, most stimulating and easiest to control). There were no significant effects of Country for Pleasure or Dominance scales. However, there was a significant main effect of Country $(F(1,46)=15.9 ; p<.001)$, and a significant interaction between Device and Country $(F(3,138)=5.5 ; p=0.001)$ for Arousal, indicating that Chinese participants were more 'excited' than UK participants overall by the new devices (although they were equally unenthused by the touchpad).

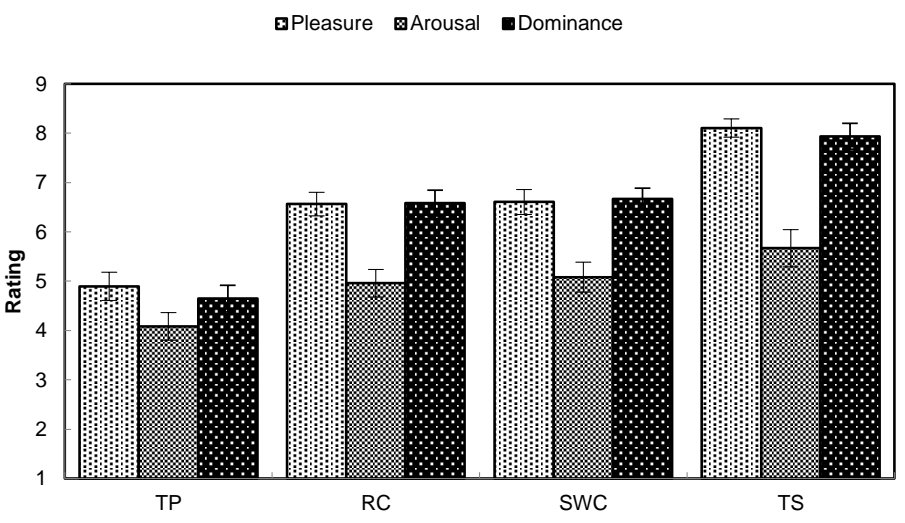

Figure 8: Emotional responses for all tasks by device, where TP = touchpad, RC = rotary controller, SWC $=$ steering wheel controls, and TS = touchscreen

\section{Preferences (Ease of Use/Interference while Driving, Overall Liking)}

There were significant main effects of Device and Country for ratings of 'ease of use while driving' (Device: $\mathrm{F}(3,135)=30.0 ; p<.001 ;$ Country: $\mathrm{F}(1,45)=10.5 ; p=.02$ ) and 'interferes with driving' (Device: $\mathrm{F}(3,135)=12.8 ; \mathrm{p}<.001 ;$ Country: $\mathrm{F}(1,45)=13.0 ; p=.01)$, with participants rating the rotary, steering controls and touchscreen as significantly easier to use while driving and interfering less with the driving task than the touchpad. In addition, the touchscreen was perceived to be easier to use than the touchpad and steering controls, but not the rotary. Overall, Chinese participants rated all devices as easier to use while driving compared to UK drivers (Figure 9). Chinese participants also indicated that that the touchscreen and steering controls, in particular, interfered less with driving. 

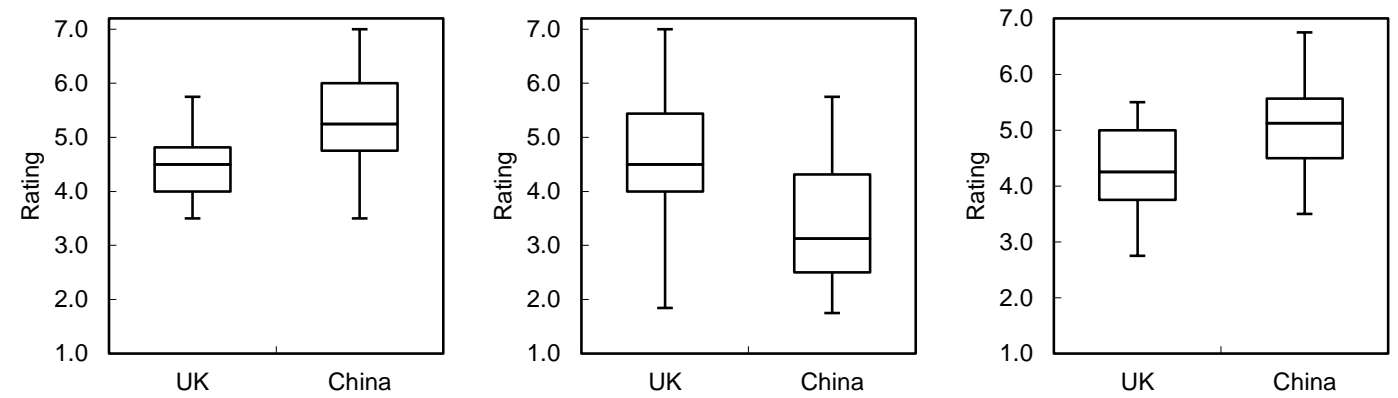

Figure 9: Comparison of subjective ratings (amalgamated for all tasks and devices) between the UK and China, showing 'ease of use' (left), 'interferes with driving' (centre) and 'overall liking' (right)

There were also significant main effects of Device $(F(3,135)=38.7 ; p<.001)$ and Country $(F(1,45)=$ 13.7; $p=0.001$ ) for ratings of 'overall liking', showing that the touchscreen was liked more than other devices, and the touchpad was liked least. Chinese participants generally liked all the devices more than their UK counterparts (Figure 9), but indicated a particular preference for the steering controls over the rotary (the opposite was true amongst UK participants).

Participants were also asked to rate their preferred device for each of the four tasks based on their experiences, by placing a marker for each device on a continuous linear scale. Positions were recorded and measured, and interpreted as a 0-100 interval scale, where a rating of ' 100 ' indicated 'best/most preferred', and ' 0 ' as 'worst/least preferred'. Ratings for each device were then compared for each task and each country. For all tasks, device ratings differed significantly in both the UK and China (Table 3).

In the UK, participants preferred the touchscreen to all other devices for the menu task. The touchpad was least preferred for this task and the rotary and steering controls were equally popular. For the list task, the touchscreen and rotary controller were significantly more popular than the touchpad and steering controls. For text and map tasks, the touchscreen was the most preferred device, with participants identifying no clear preference between the touchpad, rotary and steering controls for these tasks.

Table 3 - Significant main effects associated with device and task preferences

\begin{tabular}{|c|c|c|}
\hline Task & UK & China \\
\hline Menu Navigation & $\mathrm{F}(3,69)=29.7 ; p<.001$ & $\mathrm{~F}(3,69)=61.9 ; p<.001$ \\
\hline List Selection & $\mathrm{F}(3,69)=9.1 ; p<.001$ & $\mathrm{~F}(3,69)=20.1 ; p<.001$ \\
\hline Text Entry & $\mathrm{F}(3,69)=9.6 ; p<.001$ & $\mathrm{~F}(3,69)=23.1 ; p<.001$ \\
\hline Map Manipulation & $\mathrm{F}(3,69)=16.2 ; p<.001$ & $\mathrm{~F}(3,69)=13.8 ; p<.001$ \\
\hline
\end{tabular}


In China, the touchscreen was generally the most popular for all tasks, and the touchpad was least popular. Ratings for rotary and steering controls were generally comparable, although there were notable differences. For example, for the menu and text tasks, preferences decreased from touchscreen (which was most popular) to steering controls, then rotary and finally touchpad (least popular). The touchpad was also least popular for the list task, but there were no significant differences in ratings for the other 3 devices. For the map task, although the touchscreen was most popular, there were no significant differences between the other three devices.

When asked to identify the device that they most (and least) preferred to use in combination with the touchscreen (i.e. touchpad, rotary or steering controls), a chi-squared test revealed that more UK participants preferred the rotary controller, whereas more Chinese participants preferred the steering controls $\left(\chi^{2}=10.5 ; p<.01\right)$; the touchpad was least preferred to accompany the touchscreen by participants in both countries. Video analysis of the final, 'free-choice' drive, during which drivers were provided with the opportunity to use whichever device or devices they chose to complete each task, indicated that actual device usage was generally consistent with these preferences. However, it also provided some interesting insights regarding which devices were most suited and/or most preferred for individual task components. For example, during the list task, UK drivers commonly employed the touchscreen for locating the list, whereas the rotary was often used for moving through options. In China, while using the touchscreen was also popular to locate the list, the steering controls were more commonly employed to move through options during this task.

\section{DISCUSSION}

The study aimed to understand the effects of using alternative input devices on driver distraction and performance, and elicit preferences and provide recommendations for a secondary input device (or devices) that could be used in combination with an in-vehicle touchscreen. In addition, by conducting the study in two culturally-diverse environments, the study aimed to investigate differences in behaviour, performance and attitudes associated with the devices at a country level, which could be interpreted from a cultural perspective.

\section{HMI Evaluation}

It is evident that for most measures there were significant differences in device use and preferences within the UK and China. Objective performance measures (secondary task time, visual behaviour, driving performance) consistently revealed shortcomings associated with the touchpad - secondary tasks took the longest time to complete, it invited the most glances (many of which were longer than 2.0 seconds - a common predictor of heightened risk (NHTSA, 2013)), and TGT associated with the touchpad was significantly longer than with other devices. Using the touchpad also had the greatest impact on driving performance measures (indicated by the highest variability in speed, lateral lane position and headway). In addition, participants generally did not like the touchpad - it was associated with higher perceived workload and was identified as least pleasurable to use in both the UK ("frustrating", "slow to use") and China ("have to put in extra effort"). Nevertheless, some participants (in both the UK and China) were more positive about the touchpad: "liked the 'concept", "character recognition generally very good" (UK); "easy to draw", "writing input is easier" (China). Despite this, 
participants felt least in control when using the device, found it more difficult to use while driving, and believed that it interfered more with the driving task than other devices. The touchpad was also highlighted as least preferred for some tasks (menu and list in the UK, and for all tasks in China), although it was more favourably considered (equally as popular as the rotary and steering controls) for text and map tasks in the UK. It was also the least preferred device to use in conjunction with the touchscreen, overall, in both countries.

In contrast, the rotary and steering controls performed more favourably. In terms of secondary task performance, visual behaviour and the effect on driving, these devices were largely comparable in both countries. Subjectively, they were also popular, although it is interesting to note that after taking all devices and tasks into consideration, the rotary controller was identified as the preferred device overall to use in conjunction with the touchscreen in the UK, whereas the steering controls were the most popular secondary device in China (Chinese drivers indicated that the rotary was more 'demanding' to use than the steering controls). These preferences are reflected in comments made by participants. For example, in the UK, the rotary controller was described as; "very good for moving through list" and "comfortable to use", whereas Chinese participants described the steering controls as "more intuitive" and "more accessible when driving". Chinese drivers also described the steering controls as "more as the supplement to touchscreen", whereas they felt that the other devices "may be dominant over touchscreen".

It was also evident that in many situations using the touchscreen on its own was often quicker and required fewer off-road glances than interacting with the other devices, in line with the findings of Burnett et al. (2011). Nevertheless, MGD was notably the longest when using the touchscreen compared to the other devices, and Chinese participants made significantly longer off-road glances when using the touchscreen compared to UK participants. This may suggest that participants in both countries were more prepared to extend glances to the touchscreen, compared to other devices, or that it simply demanded more visual attention. Participant's visual behaviour while using the touchscreen may have been influenced by the location of the device, close to drivers' normal forward line of sight, i.e. drivers may have felt comfortable directing their visual attention to the touchscreen, safe in the knowledge that they could quickly return their gaze to the driving scene with minimal effort, if necessary. Indeed, improvements in lateral and longitudinal vehicle control and response times to hazardous events have been associated with reduced in-vehicle display eccentricity (Summala et al., 1996). It may also be a reflection of the fact that during the touchscreen-only condition, the device provided both control and feedback functionalities. Even so, the lowest variability in speed, headway and lateral lane position were associated with the touchscreen, suggesting that drivers in both countries were still able to maintain adequate primary task performance when using this device in the study.

The touchscreen was also identified as the most pleasurable device to use, and was described as "familiar, accurate and fast" in the UK, and in China as "good enough". Participants felt more "in control' using the touchscreen, and generally liked the device more, specifically identifying it as their preferred device for menu, text and map tasks in the UK, and for all tasks in China. These results are likely to reflect the current ubiquity of touchscreens in everyday contexts, and the broad range of potential users and use-cases (for example, see: Haßler et al., 2016; Motti et al., 2013; Joddrell and Astell, 2016), meaning that such devices are increasingly familiar and 'intuitive' to use. 
Given these results, one may conclude that using a touchscreen alone is adequate. Indeed, discounting the touchpad (which appears to be precluded as a viable candidate by drivers in both countries), the rotary and steering controls offered no apparent benefit with regard to visual demand. Nevertheless, using a touchscreen is always likely to demand some visual attention, and their expected location near to the drivers' forward line of sight intending to minimise the visual cost - may actually invite additional visual attention, and raises potential anthropometrical and operational concerns (i.e. due to the requirement to use an extended, unsupported arm during operation) - factors that were also noted by Porter et al. (2005). This could lead to potential errors and fatigue, particularly during extended periods of use. In contrast, physical devices, such as the rotary and steering controls, permit completely 'eyes-free' use (i.e. devices can be physically identified by touch and appropriate haptic cues can allow drivers to make selections without vision). This provides the freedom and flexibility to locate these devices more appropriately. For example, situating the rotary controller between driver and passenger seats, and providing an arm rest (as we did during the study), enables the driver's arm to be supported during operation. Equally, using steering controls encourages the driver to maintain their hands in contact with the steering wheel, thereby increasing vehicle stability and reducing driver workload (De Waard et al., 2010).

Drivers also relied on the touchscreen for progress and feedback when undertaking tasks - even when using the other devices - and this in itself demanded visual attention. One might reasonably expect this demand to reduce over time, as drivers become more familiar with the interface, tasks, and the operation and impact of the secondary device control actions. Moreover, secondary tasks were completed using an experimental interface, and thus no 'real-world' feedback was provided. For example, selecting the music track did not actually begin playing music. In a real-world application, such feedback would be implicit and further reduce the visual demand associated with confirming task completion, in particular.

Additionally, single device operation was enforced during the first part of the study to allow direct comparisons to be made. In reality, there are aspects of each task that may be better suited to specific devices. This is likely to reduce the overall visual burden and improve the efficiency of all tasks. For example, although behaviour and performance is generally analysed and reported at a task level, there were evidently aspects of each task that were better suited to certain devices. Indeed, when given the freedom to choose (during the final drive), the rotary controller was commonly selected in the UK during the list task to move through list options, whereas other aspects of this task (e.g. the selection of the target music track) were conducted using the touchscreen. Similar 'mixed' behaviour was observed in China, although in this case, the steering controls were a popular choice to 'step-through' list options. These observations are also reflected somewhat in the overall preferences stated by participants, with the UK delegates selecting the rotary as the device they most preferred to use in combination with the touchscreen, and the Chinese selecting the steering controls.

\section{Cultural Differences}

The study revealed some notable differences between countries regarding driving performance. For example, Chinese participants displayed greater variability in speed, headway and lane position, compared to UK drivers, suggesting differences in both longitudinal and lateral vehicle control between nations. Given that culture determines the criteria and rules, at both a formal and informal 
level, for acceptable driving styles, and may even influence driving skill (Lajunen et al., 1998), we believe that this is indicative of different cultural perspectives. Chinese participants also made longer off-road glances while undertaking the secondary tasks, and spent more time overall, with their eyes off-road than their UK counterparts. Despite this, total task times were comparable, suggesting that Chinese participants spent proportionally less time with their eyes on the road while undertaking tasks.

It is worth highlighting that different collection methods were employed to capture visual behaviour (ETG in UK and video cameras in China). Nevertheless, all data underwent manual, frame-by-frame, semantic gaze map analysis. In the UK, this was achieved using SMI BeGaze analysis software, which highlights the current fixation by presenting a target superimposed on the participant's point-of-view video. In China, two participant-facing videos streams were synchronised. From these, fixations and areas of interest were identified based on the gaze direction of the eyes - the areas of interest were sufficiently distinct in physical space to ensure that this was achievable. Moreover, the fundamental requirement during analysis was to distinguish between glances 'on-road' (mirrors, roadway etc.) and 'off-road' (the devices under consideration). Thus, although the techniques differed between countries, both required manual interpretation and allocation of areas of interest using frame-byframe video coding, and were therefore equally perspicuous for identifying and determining on/offroad glances.

As such, we remain confident that the results indicate different attitudes regarding what is acceptable at both a formal and informal level between countries and cultures, with Chinese drivers appearing to place more attention on the secondary in-vehicle devices than their UK counterparts, despite identical instructions (regarding primary/secondary task prioritisation) provided during the study. These results are consistent with Young et al. (2012), who found that Chinese drivers were more concerned by the aesthetics of in-vehicle technology designs, dismissing concerns about distraction in preference for interfaces that looked 'modern and sophisticated' and denoted a sense of high status. In contrast, UK drivers appeared to demonstrate a greater awareness of safety and the potential for distraction when interacting with the secondary in-vehicle devices during our study, although these findings could be influenced by participants' risk perception in the simulated driving environment.

It is worth noting that to facilitate tasks in each country, any text that appeared on the touchscreen was presented in the native language. Thus, in the UK, the Western-style Latin alphabet was used, whereas simplified pictogram characters were used in China. Participants were naturally required to read the text on the screen during some of the tasks. Given that this required both the extraction of visual information and comprehension of its meaning (Rayner and Pollatsek, 1989), issues of legibility (the degree to which single typographic characters can be distinguished) and readability (comprehension of the 'meaning' of the text) naturally existed. While factors such as font size and display resolution have been found to improve the legibility and readability of computer-displayed Chinese text (Huang, et al., 2009), there remains a fundamental difference between Chinese pictogram characters and the Latin alphabets used in the UK. In particular, individual Chinese characters are more visually complex and demand closer scrutiny to interpret their meaning (Radhakrishnan et al., 2015). This is likely to be reflected as an increase in 'reading' time in China, and may therefore account for the extended visual demand (exhibited by Chinese participants for some tasks) that were evident during this comparative study. 
It is also worth highlighting that although testing took place in a driving simulator in both countries, with all salient features replicated as closely as practicable between simulators, there was a notable difference in fidelity. This was due to practical limitations in time and cost associated with creating and transporting the driving simulator from the UK to China for testing. Consequently, while drivers were immersed within a real vehicle buck interior and surrounded by a 270 degrees view of the simulated scenario in the UK ('medium-fidelity'), participants were seated at a table and viewed a single screen projection of the virtual world in China ('low fidelity'). A justifiable concern is that the differences in fidelity could affect drivers' behaviour and performance, for example due to differences in risk perception. However, while there have been differences reported between real-world and simulated driving (e.g. Reed and Green, 1999), and indeed between different simulated environments within the same driving simulator (Large et al., 2015), there is evidence to suggest that differences in simulator fidelity are unlikely to have a significant effect on the metrics that we explored during the study (see: Burnett et al., 2007), particularly given that we used the same simulated environment, experimental protocols and research team to administer the test - factors that would likely have a greater impact on consistency and behaviour.

In addition, it is worth noting that there was no baseline driving condition as part of the experimental design (i.e. a drive in which participants had no secondary tasks to undertake). While this has no bearing on the primary motivation and aims behind the study, i.e. to compare the use of secondary input devices while driving in the UK and China, it could have provided additional information regarding the extent to which cultural driving norms may have influenced some of the driving performance results discussed. Nevertheless, this would have added little to visual measures, such as 'eyes-off road', given that there would no motivation to engage in a secondary in-vehicle task during a baseline drive.

A further consideration is that some of our findings and conclusions rely upon subjective data elicited using response scales. A key consideration when gathering subjective opinions from culturally-diverse groups in this manner is how different groups respond to such scales. For example, there may be strong social desirability factors influencing people's responses, with some cultures believing that there is always a 'correct' answer to a question. This has previously been observed during research conducted in China - respondents to a questionnaire exploring attitudes and opinions about China tended to give an ideal picture (in this case, what they wanted China to be) rather than describing what China actually was (Adler et al., 1989). It is also recognised that in some cultures, the context of the question asked is as important as its content. For example, in group-oriented cultures, individuals may be inclined to answer questions as if they were being addressed to the group that they belong to (in this case, 'Chinese drivers'). Conversely, in an individualistic culture, the questions will most likely be perceived and answered solely through the eyes of that individual (Hofstede, 1980).

A related concern when conducting cross-cultural research is that the research tools are equivalent in both settings (Stening and Zhang, 2007). Stening and Zhang (2007) therefore recommend that at the very least all items (participants' instructions, questionnaires etc.) should be provided to respondents in their own language, as we did during the study. However, once translated, these tools must also maintain construct validity, i.e. the questionnaires must be measuring conceptually equivalent variables in all cultures. Indeed, although we used the SAM questionnaire to measure 'emotional response' to the technology (Bradley and Lang, 1994), the questionnaires were developed in a western 
culture and therefore, although these were carefully translated (with translations independently checked and verified), they may not have measured equivalent constructs, or equivalent constructs may not actually exist, in China (Stening and Zhang, 2007). In addition, Chen (1995) noted the relative reluctance of Chinese people to disclose their thoughts and express their opinions when participating in research, and this will naturally impact on the collection of subjective data. In a culture such as China, for example, 'face-to-face' methods (handled "sensitively") have been found to be more likely to yield reliable results (Stening and Zhang, 2007). It is primarily for this reason that ratings were taken verbally in China by a local Chinese collaborator, rather than asking participants to record their own written responses, as was the case in the UK. This also helped to ensure anonymity - another consideration that may have a major impact on cooperation and reliable results elicitation in China (Stening and Zhang, 2007).

Overall, we recognise that there is substantial evidence showing that undertaking cross-cultural research (especially where this is outside the researcher's cultural background and experience) presents a number of methodological challenges (Stening and Zhang, 2007). Nevertheless, by using the perspectives, insights and skills of a well-qualified local collaborator, as we did, methodological and practical concerns can be mitigated and/or managed. Even so, in presenting, interpreting and discussing the results of the study, we have remained mindful of the potential pitfalls (conceptual and metric inequivalence, biased data etc.), and also the potential impact of our own cultural values and attitudes: the 'researcher effect' is well-recognised in the social sciences, but thought to be especially prevalent in cross-cultural research (Stening and Zhang, 2007). We have therefore attempted to highlight if and where compromises or assumptions may have been made, and have been careful to avoid carelessly applying western theories in search of Chinese realities (Pye, 1992).

\section{CONCLUSION}

The study evaluated secondary input devices to support an automotive touchscreen user interface. By conducting the investigation in both the UK and China, it also explored cultural differences associated with the use of these devices. The study revealed that overall the touchscreen was a popular solution in both the UK and China - it was the most preferred and least demanding device to use according to participants (although these opinions may have been influenced by the familiarity and ubiquity of such devices in non-automotive domains). In contrast, the touchpad was least preferred, most demanding and somewhat ineffective for the tasks evaluated during the study. Nevertheless, there were aspects of each task that appeared better suited to different devices and the rotary and steering controls (which were largely comparable across most measures) both presented as viable solutions, and are therefore worthy candidates for further investigations. Of particular interest is that UK drivers expressed a preference for the rotary to be used in conjunction with the touchscreen, whereas the steering controls were favoured by the Chinese. Indeed, both devices offer a variety of input techniques (enabling multiple functionality), and can be located to discourage visual attention while ensuring that the driver's arm is supported during operation. A particular advantage to the steering controls is that they encourage the driver to maintain their hand in contact with (or in very close proximity to) the steering wheel, thereby providing support and stabilisation. 
The study also revealed noteworthy differences between the UK and China more generally, particularly with regard to driving performance and visual behaviour, with results indicating that Chinese drivers (who were generally more 'excited' by the novelty of the technology under investigation) dedicated more time and visual attention to the devices, apparently at the expense of their driving performance. Even so, these drivers rated the devices as easier to use and stated that they interfered less with their driving.

In addition to providing general guidance, the study demonstrates the importance of considering a local cultural perspective during the design and evaluation of novel in-vehicle devices, although it is acknowledged that cultural factors are complex and interrelated, and caution should be applied if attempting to draw detailed cultural design guidance or recommendations from the work. Further work should therefore explore the devices in greater depth and during extended periods of testing, and aim to validate findings within diverse cultural settings. In addition, given that the study was conducted in a driving simulator, there is a need to consider real-world factors, such as the effect of road and vehicle vibrations on operational performance and accuracy, and the impact that these factors have on visual behaviour and subjective preferences in both nations.

\section{ACKNOWLEDGEMENTS}

The research was conducted in collaboration with Jaguar Land Rover Research (UK) and the authors would like to gratefully acknowledge their support. We would also like to thank Acorn Marketing and Research Consultants (China), and the University of Nottingham Ningbo Campus (UNNC), who provided local expertise, facilities and support in China.

\section{REFERENCES}

Adell, E., 2010. Acceptance of driver support systems. In Proceedings of the European Conference on Human Centred Design for Intelligent Transport Systems. Berlin, Germany.

Adler, N.J., Campbell, N. and Laurent, A., 1989. In search of appropriate methodology: From outside the People's Republic of China looking in.Journal of international business studies, pp.61-74.

Ahmad, BI and Murphy, JK and Godsill, S and Langdon, PM and Hardy, R (2017) Intelligent Interactive Displays in Vehicles with Intent Prediction: A Bayesian framework. IEEE Signal Processing Magazine, 34. pp. $82-94$

Allison, R., 1995. Cross-cultural factors in global advertising. Cross Culture-Interkulturelles Handeln in der Wirtschaft.-Sternenfels, Berlin: Verl. Wiss. und Praxis, pp.92-101.

Aslan, I., Krischkowsky, A., Meschtscherjakov, A., Wuchse, M., Tscheligi, M., 2015. A leap for touch: proximity sensitive touch targets in cars. AutoUI2015 Conference, pp. 39-46. ACM.

Bradley, M.M., Lang, P.J., 1994. Measuring emotion: the self-assessment manikin and the semantic differential. Journal of behavior therapy and experimental psychiatry, 25(1), pp.49-59.

Brookhuis, K., Waard, D.D. and Mulder, B., 1994. Measuring driving performance by car-following in traffic. Ergonomics, 37(3), pp.427-434. 
Burnett, G., Crundall, E., Large, D., Lawson, G., Skrypchuk, L., 2013. A study of unidirectional swipe gestures on in-vehicle touch screens. AutoUI2013 Conference, pp. 22-29. ACM

Burnett, G., Lawson, G., Millen, L., Pickering, C. 2011. Designing touchpad user-interfaces for vehicles: which tasks are most suitable? Behaviour \& Information Technology, 30(3), 403-414.

Burnett, G.E., Irune, A. and Mowforth, A., 2007. Driving simulator sickness and validity: how important is it to use real car cabins?. Advances in transportation studies, 2007(Spec Iss), pp.33-42.

Chen, G.M., 1992. Differences in self-disclosure patterns among Americans versus Chinese: A comparative study, Journal of Cross-Cultural Psychology 26(1): 84-91.

Couch, A. and Keniston, K., 1960. Yeasayers and naysayers: agreeing response set as a personality variable. The Journal of Abnormal and Social Psychology, 60(2), p.151-174

De Waard, D., Van den Bold, T.G. and Lewis-Evans, B., 2010. Driver hand position on the steering wheel while merging into motorway traffic. Transportation research part F: traffic psychology and behaviour, 13(2), pp.129-140.

Eren, A.L., Burnett, G., Large, D.R., 2015a. Can in-vehicle touchscreens be operated with zero visual demand? An exploratory driving simulator study. In DDI2015 Conference.

Eren, A.L., Burnett, G., Thompson, S., Harvey, C., Skrypchuk, L. 2015b. Identifying a set of gestures for in-car touch screens. IEHF Conference

Gibson, R., 2002. Intercultural Business Communication: An Introduction to the Theory and Practice of Intercultural Business Communication for Teachers, Language Trainers, and Business People. Oxford University Press.

Goszczyńska, M. and Rosłan, A., 1989. Self-evaluation of drivers' skill: A cross-cultural comparison. Accident Analysis \& Prevention, 21(3), pp.217-224.

Hart, S.G., Staveland, L.E., 1988. Development of NASA-TLX (Task Load Index): Results of empirical and theoretical research. Advances in psychology, 52, pp.139-183

Haßler, B., Major, L. and Hennessy, S., 2016. Tablet use in schools: A critical review of the evidence for learning outcomes. Journal of Computer Assisted Learning, 32(2), pp.139-156.

Hofstede, G. (1980) Culture's Consequences: International Differences in Work-Related Values. Sage Publications, Beverly Hills.

Horberry, T., Regan, M.A. and Stevens, A. eds., 2017. Driver acceptance of new technology: Theory, measurement and optimisation. CRC Press.

Huang, D.L., Rau, P.L.P. and Liu, Y., 2009. Effects of font size, display resolution and task type on reading Chinese fonts from mobile devices. International Journal of Industrial Ergonomics, 39(1), pp.81-89.

ISO 15007-1:2014. 2014 Road vehicles - Measurement of driver visual behaviour with respect to transport information and control systems - Part 1: Definitions and parameters

Jamson, S., 2010. Acceptability data-what should or could it predict?. In: International seminar on acceptance, Paris, France. 
Joddrell, P. and Astell, A.J., 2016. Studies involving people with dementia and touchscreen technology: a literature review. JMIR rehabilitation and assistive technologies, 3(2).

Kerlinger, F.W. (1973) The Foundations of Behavioral Research, 2nd edn. New York: Holt, Rinehart \& Winston.

Khaslavsky, J., 1998, April. Integrating culture into interface design. In CHI 98 Cconference Summary on Human Factors in Computing Systems (pp. 365-366). ACM.

Knapp, B., 2007. Mental models of Chinese and German users and their implications for MMI: experiences from the case study navigation system. Human-Computer Interaction. Interaction Design and Usability, pp.882-890.

Lajunen, T., Corry, A., Summala, H. and Hartley, L., 1998. Cross-cultural differences in drivers' selfassessments of their perceptual-motor and safety skills: Australians and Finns. Personality and Individual Differences, 24(4), pp.539-550.

Lamble, D., Laakso, M., Summala, H., 1999. Detection thresholds in car following situations and peripheral vision: implications for positioning of visually demanding in-car displays. Ergonomics 42 , 807-815.

Large, D. R., Burnett, G. E., Lawson, G., Crundall, E. De-Kremer, S. 2013. Measuring the distraction of alternative list-scrolling techniques when using interactive touchscreen displays in vehicles. DDI2013

Large, D. R., Burnett, G., Mohd-Hasni, Y. 2017. Capturing Cultural Differences between UK and Malaysian Drivers to Inform the Design of In-Vehicle Navigation Systems. International Journal of Automotive Engineering, 8(3), pp.112-119

Large, D.R., van Loon, E., Burnett, G. and Pournami, S., 2015, September. Applying NHTSA task acceptance criteria to different simulated driving scenarios. In Proceedings of the 7th International Conference on Automotive User Interfaces and Interactive Vehicular Applications (pp. 117-124). ACM.

Motti, L.G., Vigouroux, N. and Gorce, P., 2013, November. Interaction techniques for older adults using touchscreen devices: a literature review. In Proceedings of the 25th Conference on I'Interaction Homme-Machine (p. 125). ACM.

NHTSA. 2013. Visual-Manual NHTSA Driver Distraction Guidelines For In-Vehicle Electronic Devices. NHTSA-2010-0053

Olaverri-Monreal, C. and Bengler, K.J., 2011, June. Impact of cultural diversity on the menu structure design of Driver Information Systems: a cross-cultural study. In Intelligent Vehicles Symposium (IV), 2011 IEEE (pp. 107-112). IEEE.

Özkan, T., Lajunen, T., Chliaoutakis, J.E., Parker, D. and Summala, H., 2006. Cross-cultural differences in driving behaviours: A comparison of six countries. Transportation research part F: traffic psychology and behaviour,9(3), pp.227-242.

Pitts, M., Burnett, G.E., Skrypchuk, Lee, Wellings, T., Attridge, A., Williams, M.A. (2012). Visual-haptic feedback interaction in automotive touchscreen use, Displays, 33(1), 7-16 
Porter, J.M., Summerskill, S.J., Burnett, G.E. and Prynne, K., 2005, August. BIONIC-'eyes-free'design of secondary driving controls. In Proc. of the Accessible Design in the Digital World Conference. Dundee, $U K$.

Pye, L.W., 1992. Social science theories in search of Chinese realities. The China Quarterly, 132, pp.1161-1170.

Radhakrishnan, H., Hartwig, A., Charman, W.N. and Llorente, L., 2015. Accommodation response to Chinese and Latin characters in Chinese-illiterate young adults. Clinical and Experimental Optometry, 98(6), pp.527-534.

Reed, M.P. and Green, P.A., 1999. Comparison of driving performance on-road and in a low-cost simulator using a concurrent telephone dialling task. Ergonomics, 42(8), pp.1015-1037.

Roy, A., Walters, P.G. and Luk, S.T., 2001. Chinese puzzles and paradoxes: Conducting business research in China. Journal of Business Research,52(2), pp.203-210.

Schade, J. and Schlag, B., 2003. Acceptability of urban transport pricing strategies. Transportation Research Part F: Traffic Psychology and Behaviour, 6(1), pp.45-61.

Stening, B.W. and Zhang, M.Y., 2007. Methodological challenges confronted when conducting management research in China. International Journal of Cross Cultural Management, 7(1), pp.121142.

Summala, H., Nieminen, T. and Punto, M., 1996. Maintaining lane position with peripheral vision during in-vehicle tasks. Human factors, 38(3), pp.442-451.

Young, K.L., Rudin-Brown, C.M., Lenné, M.G. and Williamson, A.R., 2012. The implications of crossregional differences for the design of In-vehicle Information Systems: A comparison of Australian and Chinese drivers. Applied Ergonomics, 43(3), pp.564-573.

Zwahlen, H.T., Adams, C.C., Debald, D.P. 1988. Safety aspects of CRT touch panel controls in automobiles. In A.G. Gale et al. (Eds.), Vision in vehicles II (pp. 335-344). Amsterdam: Elsevier Science Publishers B.V. 


\section{Appendix - Driver Acceptance Rating Scales}

Please rate your experience using the device, during the last task only, by marking the point on the scales below which best describes your level of agreement with statements.

The device was...

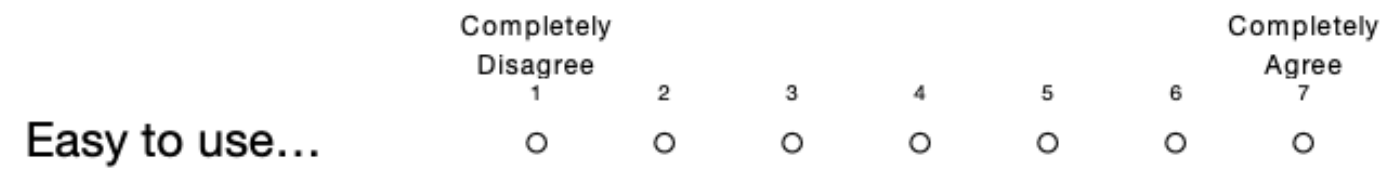

Pleasurable to use...

Easy to learn...

$\circ \quad \circ \quad 0 \quad 0$

$\circ 0$

○

I could manipulate the device...

\begin{tabular}{|c|c|c|c|c|c|c|c|}
\hline & $\begin{array}{l}\text { Completely } \\
\text { Disagree }\end{array}$ & & & & & & $\begin{array}{l}\text { ompletely } \\
\text { Agree }\end{array}$ \\
\hline & & 2 & ${ }^{3}$ & 4 & 5 & 6 & \\
\hline Accurately... & 0 & 0 & 0 & o & 0 & 0 & 0 \\
\hline
\end{tabular}

Comfortably... $\quad \circ \quad \circ \quad \circ \quad \circ \quad \circ \quad \circ \quad \circ$

$\begin{array}{llllllll}\text { Easily... } & \circ & \circ & \circ & \circ & \circ & \circ & \circ\end{array}$

$\begin{array}{llllllll}\text { Quickly... } & \circ & \circ & \circ & \circ & \circ & \circ & \circ\end{array}$

$\begin{array}{llllllll}\text { Confidently... } & \circ & \circ & \circ & \circ & \circ & \circ & \circ\end{array}$

What is your rating of this device based on the last task?

$\begin{array}{ccccccc}\text { Hate it } & & & & & \text { Love it } \\ 1 & 2 & 3 & 4 & 5 & 6 & 7 \\ 0 & 0 & 0 & 0 & 0 & 0 & 0\end{array}$


Please rate your experience using the device, during all tasks while driving, by marking the point on the scales below which best describes your level of agreement with statements.

When driving, the device...

\begin{tabular}{|c|c|c|c|c|c|c|c|}
\hline \multicolumn{7}{|c|}{ Completely } & \multirow{2}{*}{$\begin{array}{c}\text { Completely } \\
\text { Agree }\end{array}$} \\
\hline & $\underset{1}{\text { Disagree }}$ & 2 & 3 & 4 & 5 & 6 & \\
\hline Was easy to use... & 0 & 0 & 0 & 0 & 0 & $\mathrm{o}$ & 0 \\
\hline
\end{tabular}

Did not interfere... $\circ \quad \circ \quad \circ \quad \circ \quad \circ \quad \circ \quad \circ$ with driving

What is your overall rating of this device based on your experiences using it while driving?

$\begin{array}{ccccccc}\text { Hate it } & & & & & & \text { Love it } \\ 1 & 2 & 3 & 4 & 5 & 6 & 7 \\ 0 & 0 & 0 & 0 & 0 & 0 & 0\end{array}$

